\title{
Impact of SSKI vs. Corticosteroids on Total Thyroidectomy for Graves Disease: A Retrospective Review
}

\author{
Timothy C. Baumgartner ${ }^{1}$, Hannah R. Allison², Alexandria D. McDow² \\ ${ }^{1}$ Indiana School of Medicine, ${ }^{2}$ Indiana School of Medicine, Department of Surgery²
}

Background: Graves disease is an autoimmune disorder resulting in overproduction of thyroid hormone. While medical management can be used to reestablish euthyroid state, definitive management includes radioactive iodine or total thyroidectomy. For patients undergoing thyroidectomy, SSKI is frequently prescribed preoperatively to decrease thyroid vascularity and intraoperative blood loss. However, corticosteroids is often given when rapid preparation is necessary prior to surgery. This study explores the impact of SSKI compared to corticosteroids and hypothesizes that replacement of SSKI with corticosteroid will improve surgical outcomes.

Methods: We performed a retrospective review of 26 Graves patients undergoing total thyroidectomy from 2015-2020. 18 patients received SSKI prior to surgery, 2 received a corticosteroid in addition to SSKI, 1 received a corticosteroid alone, and 5 received neither. Symptomatology, length of stay, intraoperative blood loss, transient hypoparathyroidism, and thyroid weight were compared.

Results: Patients receiving corticosteroids experienced shorter stays (1.0 day vs 1.3 days), greater postoperative parathyroid hormone levels $(43.0 \mathrm{pg} / \mathrm{mL}$ vs $28.9 \mathrm{pg} / \mathrm{mL}$ ), less intraoperative blood loss $(14.4 \mathrm{~mL}$ vs $41.4 \mathrm{~mL})$, and presented with smaller thyroid glands $(30 \mathrm{~g}$ vs $47 \mathrm{~g}$ ) compared to those who received SSKI. There was a statistically significant difference in estimated blood loss between the groups $(p=0.0348)$. For some patients, the addition of a steroid to SSKI decreased surgery time (1.98 hours vs 2.32 hours).

Conclusion: Despite limitations in sample size and the retrospective nature of this study, results suggest a positive impact of corticosteroid use on surgical and patient outcomes compared to SSKI alone. Future examination may benefit from greater sample size and equal distribution of patients among groups. We propose further exploration with a prospective study to evaluate the impact of SSKI vs. corticosteroids on surgical difficulty and postoperative complications for Graves disease patients. 